\title{
Two-dimensional electron density in the solar corona from inversion of white light images - Application to SOHO/LASCO-C2 observations
}

\author{
E. Quémerais ${ }^{1}$ and P. Lamy ${ }^{2}$ \\ 1 Service d'Aéronomie du CNRS, BP 3, 91371, Verrières-le-Buisson, France \\ 2 Laboratoire d'Astronomie Spatiale, BP 8, 13376 Marseille Cedex 12, France \\ Received 22 March 2002 / Accepted 28 June 2002

\begin{abstract}
We present here the inversion method which has been developed to derive the electron density in the Solar Corona from LASCO-C2 white light images. We discuss how our inversion scheme can be applied either to images of polarized brightness of the corona or images of total brightness after removal of the dust and stray light components. The outputs from both schemes are compared. For the inversion of two-dimensional images of the solar corona, it is necessary to assume a global geometry for the electron density. We compare results obtained for spherical and axi-symmetric geometries. Finally, our results for December 1996 are compared to previous derivations of the electron radial density profiles obtained by other measurements.
\end{abstract}

Key words. Sun: corona - Sun: general - scattering

\section{Introduction}

The derivation of the electron density in the solar corona from its white-light polarized radiance $p B$ is a classical problem of coronal physics first addressed by Minnaert (1930) and Van de Hulst (1950). Starting from the expression of the Thompson scattering by a single electron, taking into account the fact that the K-corona is optically thin and assuming a power-law representation for the polarized radiance as a function of the apparent height from the Sun center, Van de Hulst derived a similar parametric representation for the electron density $N_{\mathrm{e}}(r)$ as a function of the radial distance from the Sun center. This method has been widely applied to obtain radial profiles of the coronal electron density from calibrated, white-light images along selected radial directions. Well-known examples include for example, Allen (1973), Munro \& Jackson (1977), Crifo-Magnant \& Picat (1980) and quite recently, Cranmer et al. (1999) to analyze observations obtained with the whitelight channel of the SOHO/UVCS experiment.

The first attempts to obtain two-dimensional maps of the electron density were performed by assuming that $p B$ and $N_{\mathrm{e}}$ can be represented by the product of separate functions, one of the radial distance $r$ and the other of latitude $\lambda$ producing an axisymmetric model (e.g., Saito 1970; Munro \& Jackson 1977). More general solutions have been sought by expressing the electron density as a sum of Legendre polynomials, an approach pioneered by Altschuler \& Perry (1972) and pursued

Send offprint requests to: E. Quémerais, e-mail: eric.quemerais@aerov.jussieu.fr by Gabryl et al. (1999), or by implementing arithmetic reconstruction techniques (Wilson 1976).

In this article, we present a new method for inverting radiance images of the corona which we specifically developed to retrieve 2-dimensional maps of the electron density from the images obtained with the SOHO/LASCO coronographs (Brueckner et al. 1995) taking advantage of its excellent radiometric performances. The inversion proceeds along radial directions and assumes a standard radial variation of the electron density as a power law. The radiance is then calculated and a multiplicative correction factor is iteratively applied to the electron density until the calculated radiance satisfactorily matches the observed one. This procedure is valid since the white light corona is optically thin. Since the distribution of electron in the corona is in reality a 3-dimensional function, the analysis of projected 2-dimensional images requires an an assumption on the distribution of electrons along the line-ofsight. We consider two simple geometries that we feel reflect the idealized extreme cases of the corona during a solar cycle. The assumption of local spherical geometry is appropriate to a corona of the maximum type while that of global axi-symmetry better corresponds to a corona of the minimum type. We first apply our inversion technique to polarized radiance $p B$ images but we also discuss how it can be applied to total radiance images once the F-corona and stray light components have been removed (Lamy et al. 1997). We present maps and radial profiles of the electron density retrieved from LASCO-C2 images obtained in December 1996, close to the period of the last solar minimum and compare them with published results. 


\section{Calculation of the electron density}

\subsection{Thompson scattering in the solar corona}

The equations required for the calculation of the electron density were established by Minaert (1930), Van de Hulst (1950) and Billings (1966). The K-corona radiance $B_{\mathrm{k}}$ is split into its tangential and radial components $B_{\mathrm{k}}=I_{\mathrm{t}}+I_{\mathrm{r}}$. The polarized radiance $p_{\mathrm{k}} B_{\mathrm{k}}=I_{\mathrm{t}}-I_{\mathrm{r}}$ and $I_{\mathrm{t}}$ are given by the following integrals along the line-of-sight:

$$
\begin{aligned}
& I_{\mathrm{t}}-I_{\mathrm{r}}=\frac{B_{\odot}}{1-\frac{u}{3}} \frac{\pi \sigma}{2} \int_{\mathrm{LOS}}[(1-u) A(r)+u B(r)] \frac{\rho^{2}}{r^{2}} N e(s) \mathrm{d} s \\
& I_{\mathrm{t}}=\frac{B_{\odot}}{1-\frac{u}{3}} \frac{\pi \sigma}{2} \int_{\operatorname{LOS}}[(1-u) C(r)+u D(r)] N e(s) \mathrm{d} s
\end{aligned}
$$

Using the notations of Fig. 1a, $\rho=O P$ is the perpendicular distance between the LOS and Sun center O (i.e., the impact parameter), $s=P M$ is the distance between $\mathrm{P}$ and any point $\mathrm{M}$ of the LOS and $r=O M$ is the radial distance. These distances are related by $r^{2}=s^{2}+\rho^{2}$ so that $\mathrm{d} s=\frac{r \mathrm{~d} r}{\sqrt{r^{2}-\rho^{2}}}$.

$B_{\odot}$ is the mean solar radiance, $u$ is the linear limb-darkening coefficient, $\sigma=6.65 \times 10^{-25} \mathrm{~cm}^{2}$ is the Thompson scattering cross-section for a single electron and the four functions $A, B, C$ and $D$ are given by

$$
\begin{aligned}
& A(r)=\cos \Omega \sin ^{2} \Omega \\
& B(r)=-\frac{1}{8}\left[1-3 \sin ^{2} \Omega-\cos ^{2} \Omega\left(\frac{1+3 \sin ^{2} \Omega}{\sin \Omega}\right) \ln \left(\frac{1+\sin \Omega}{\cos \Omega}\right)\right] \\
& C(r)=\frac{4}{3}-\cos \Omega-\frac{\cos ^{3} \Omega}{3} \\
& D(r)=\frac{1}{8}\left[5+\sin ^{2} \Omega-\cos ^{2} \Omega\left(\frac{5-\sin ^{2} \Omega}{\sin \Omega}\right) \ln \left(\frac{1+\sin \Omega}{\cos \Omega}\right)\right]
\end{aligned}
$$

The angle $\Omega$ is defined by $\sin \Omega=R_{\odot} / r$ and $\cos \Omega=$ $\sqrt{1-\sin ^{2} \Omega}$ where $R_{\odot}$ denotes the solar radius.

The general, 3-dimensional function representing the real distribution of electrons in the corona cannot be simply retrieved from the analysis of projected 2-dimensional images of the radiance. Because the radiance values can be separated into a function of distance and position angle, we can recover a two-dimensional function for the electron density if we assume some simple geometry for the distribution of electrons along the LOS. We consider below two classical geometries, often considered as approximately representative of the $e x$ treme types of the corona, spherical symmetry for the maximum type and axial symmetry for the minimum type. Note that these symmetries need not be global but only local as will become clear in the section below.

\subsection{Inversion in the case of spherical symmetry}

The inversion of either the polarized or the total radiance images proceeds along radial directions, from the outer limit of the field-of-view (FOV) towards the center of the Sun. We start with a standard power-law representation of the electron density $N_{\mathrm{e}}(r)$ appropriate to the outer part of the FOV. In the case of the LASCO-C2 images, this corresponds to the range 5 to $6 R_{\odot}$ and a one-term expression is adequate so that, for the $i$ th radial direction, we have

$N_{\mathrm{e}}(r)=a_{i} \cdot r^{b_{i}}$.

It is thus required that the spherical symmetry of $N_{\mathrm{e}}(r)$ only holds locally, i.e., in each half-plane $O P M$ defined by the radial direction $O P$ of index $i$. To start with, a common value of the $b_{i} \mathrm{~s}$ is taken from a standard model, e.g., Allen (1973). The corresponding radiance is calculated in the same radial range by integrating the electron along the LOS. Note that the integral extends beyond $6 R_{\odot}$ to obtain a correct estimate. The slope of the calculated radiance is then compared to the data and the value of $b_{i}$ is iteratively adjusted until a satisfactory fit is reached. This step by step correction of the electron density is justified by the fact that the white-light corona is optically thin. The coefficient $a_{i}$ is then simply calculated by dividing the data by the computed radiance. This solution for $N_{\mathrm{e}}(r)$ is then used as a first estimate in the neighboring interval of radial distance and the adjustment proceeds as described above. The operation is repeated until the inner limit of the FOV is reached. A two-dimensional map of the electron density is finally obtained by repeating the above procedure for all radial directions, typically 360 . At the end, the calculated radiance map is compared with the data to check the quality of the inversion. Several tests have proved that this procedure is very robust.

\subsection{Inversion in the case of axial symmetry}

In this section, we assume that the solar corona has an axis of symmetry passing through the center of the Sun. For our present applications, we have chosen the solar rotation axis but choosing any other symmetry axis will not affect the procedure. The geometry is shown in Fig. 1b. We can define a cylindrical reference system $(x, \alpha, z)$ where $x=A M$ is the distance of any point of the LOS to the axis, $z=O A$ is the height of the LOS, and $\alpha$ is the angle defined by the directions $A P$ and $A M$. The equatorial plane is defined by $z=0$ and the plane of the sky, by $\alpha=0$ and $\alpha=\pi$.

The inversion of either the polarized or total radiance proceeds independently for the two (east and west) hemispheres, along lines of constant height, starting from the edge of the FOV up to the axis. It is only required that the axial symmetry holds locally, i.e., in each half-plane $A P M$, either east or west, defined by the height $z$. The electron density calculated in the two hemispheres may not necessarily match and a discontinuity may appear along the axis of symmetry. This could be inconvenient and we implemented a scheme where the electron density at point $M(x, \alpha, z)$ is a weighted function of the densities found on the two sides, $N e_{1}$ at point $(x, 0, z)$ and $N e_{2}$ at point $(x, \pi, z)$ via:

$N e=N e_{1} \cdot \cos ^{2} \alpha+N e_{2} \cdot \sin ^{2} \alpha$.

Any discontinuity therefore disappears but the assumption of local axi-symmetry no longer strictly applies and we call this case "quasi axi-symmetry". 


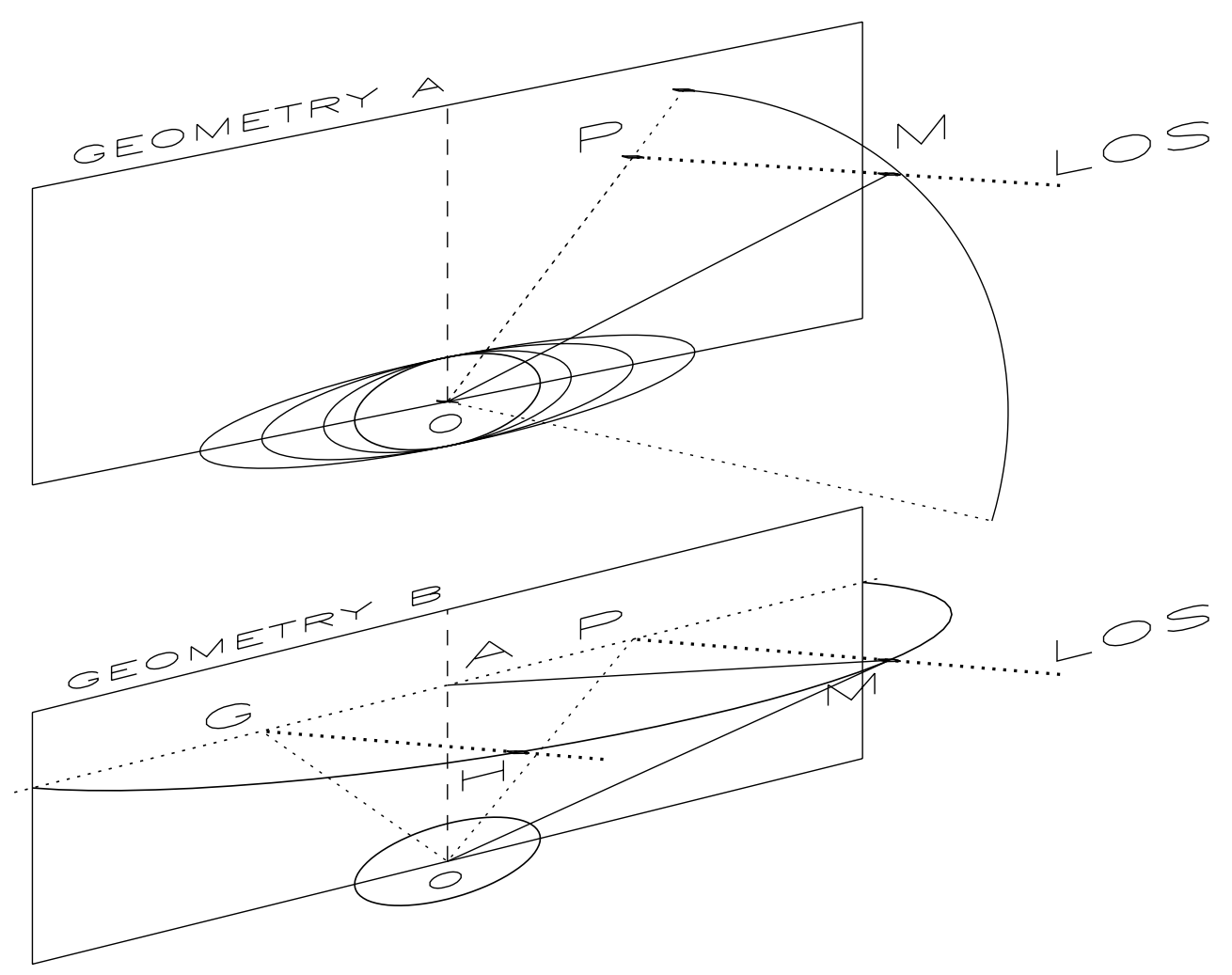

Fig. 1. Illustrations and notations for the two considered geometries. Top panel: spherical symmetry. Bottom panel: axial symmetry. Top panel (1a) The notations used in Eqs. (1) and (2) are: $r=O M, \rho=O P$ and $s=P M\left(r^{2}=\rho^{2}+s^{2}\right)$. Bottom panel (1b) the angle $\alpha$ is defined by $(\widehat{P A M})$.

The inversion starts with the electron density map initially obtained under the assumption of spherical symmetry. We apply an iterative scheme similar to that developed in the above section. Once again we compute the radiance values assuming that densities are correct for heights larger than the considered impact parameter. Then by comparing with the data, we derive a correction term for the density. The iterative process is performed along a line of constant $z$ and simultaneously on both sides of the symmetry axis as illustrated in Fig. 1 by the two lines $P M$ and $G H$.

\subsection{Inversion of the white light total radiance images}

It may sometimes be useful to obtain the electron density from total radiance K-corona images. Potential applications are the full resolution LASCO-C2 images (the $p B$ images are generally half resolution) and the $\mathrm{C} 3$ images for which the classical assumption that the polarization of the F-corona is zero becomes questionable. As already pointed out, the two algorithms described in the previous section apply as well to the inversion of total radiance images. An initial version of this application was published by Lamy et al. (1997). This technique was also used by Hayes et al. (2001) to derive density profiles as observed during the February 1998 eclipse.

The two inversions are not strictly equivalent. This can be shown by a comparison of the relative weight functions along the line-of-sight for various impact parameters. Let us rewrite the equations for the total radiance $B_{k}$ and the polarized radiance $p B$ as follows:

$$
\begin{aligned}
& B_{k}=\int_{\operatorname{LOS}} N_{\mathrm{e}}\left(\sqrt{s^{2}+\rho^{2}}\right) F(s, \rho) \mathrm{d} s \\
& p B=\int_{\operatorname{LOS}} N_{\mathrm{e}}\left(\sqrt{s^{2}+\rho^{2}}\right) G(s, \rho) \mathrm{d} s .
\end{aligned}
$$

In Fig. 2, we show a comparison of the two weight functions $F(s, \rho)$ and $G(s, \rho)$ for two values of the impact parameter $\rho$ that are relevant to the LASCO-C2 field of view, 3 and $6 R_{\odot}$. The weight functions have been normalized to allow for comparison. We see that the weight distribution along the LOS is more concentrated near the point of minimum solar distance for $p B$. This means that the inversion procedure will be more efficient when applied to the polarized brightness because points at larger distances from the Sun have a smaller relative contribution than in the case of the total brightness. In other words, the determination of the density in the plane of the sky is less influenced by the density distribution along the line of sight. Although the weight function gets less and less peaked with increasing values of $\rho$, the $p B$ weight function is always sharper than in the case of $B_{\mathrm{k}}$.

\section{Application to the LASCO-C2 images}

The purpose of this section is to illustrate the application of our method to the LASCO-C2 images, to discuss the influence of the various assumptions and compare to published results 


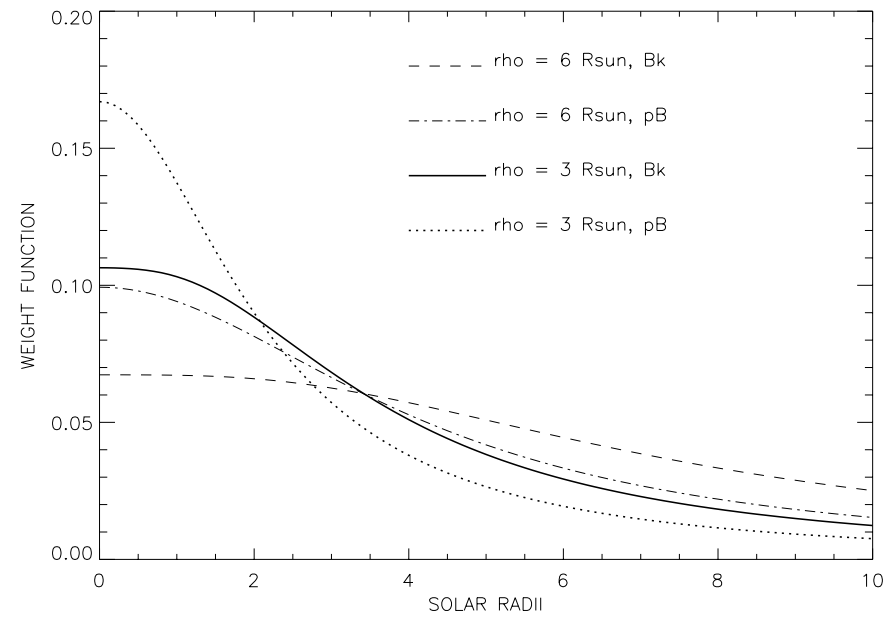

Fig. 2. The weight functions $F$ and $G$ are plotted as a function of distance from the plane of the sky along the LOS for two values of the impact parameter $\rho$. Note that the weight functions for $p B$ have narrower peaks than that for $B_{k}$.

on the electron density. We have already analyzed 4 years of LASCO-C2 $p B$ data to generate synoptic maps of the electron density from February 1996 to February 2000 and study the influence of the solar cycle (Llebaria et al. 1999; Lamy et al. 2002).

Here we consider the month of December 1996 since Cranmer et al. (1999) have already published $N_{\mathrm{e}}$ data from the white-light channel of UVCS. This month was very near the minimum of activity of solar cycle 22 . The LASCO-C2 polarized radiance images $p B$ were generated from the daily polarization sequences consisting a set of three polarized images taken through the orange filter $(540-640 \mathrm{~nm})$ and polarizers oriented at $60^{\circ}, 0^{\circ}$ and $-60^{\circ}$. The total radiance images were obtained as described by Lamy et al. (1997).

\subsection{Inversion of polarized versus total radiance images}

To perform this comparison, we consider data obtained on 29 December, 1996. The total radiance image of the K-corona is displayed in Fig. 3 and the $N_{\mathrm{e}}$ resulting from its inversion in Fig. 4. Rather than showing the corresponding figures for the polarized radiance, we present the pixel-to-pixel scatter plot of the two $N_{\mathrm{e}}$ images: the electron density obtained from the $B_{\mathrm{k}}$ image is plotted as a function of the density obtained from the $p B$ image. Figure 5 indicates an excellent agreement between the two results.

We perform a final check by calculating the total radiance from the electron density map obtained from the $p B$ data and comparing it to the original K-corona image. The excellent agreement is illustrated by the pixel-to-pixel scatter plot displayed in Fig. 6.

In what follows, we only consider results from the inversion of $p B$ images. The first reason is that this give access to a more localized information as discussed (Fig. 2). The second reason is that it is almost standard practice for ground-based observations (e.g. the Mark III and IV coronagraphs).

\subsection{Spherical or cylindrical symmetry?}

For this study, we averaged 22 daily observations obtained in December 1996. The resulting $p B$ image (Fig. 7) is less affected by transient features and displays an average state for the solar corona during this month, close to the minimum of activity. It clearly exhibits an axial symmetry with its axis close to the rotation axis of the Sun. Figure 8 displays the $N_{\mathrm{e}}$ map from the inversion of this average $p B$ image assuming spherical symmetry. We note that this assumption does not really perturb the spatial distribution as the $N_{\mathrm{e}}$ map preserves the axi-symmetry of the $p B$ image. We next proceed by performing the inversion in the case of "strict", local axi-symmetry and display the ratio of the $N_{\mathrm{e}}$ image to the one obtained above (spherical symmetry) in Fig. 9. Not surprisingly, there appears a discontinuity along the axis, most pronounced at the north pole. This can be alleviated by introducing the quasi axi-symmetry approximation and the result is shown in Fig. 10. Apart from the discontinuity, it is not very different from the previous result because the two sides of the average $p B$ image are not too different. This will not be true for daily observations with a strong asymmetry around the rotation axis of the Sun.

As expected, the three methods give similar results in the equatorial region (and have strictly the same along the equatorial direction) because the different assumptions there are quasi equivalent. The discrepancies appear with increasing latitudes but not in a monotonic way. The axial solution is depressed compared with the spherical solution at mid-latitudes $\left(\sim 45^{\circ}\right)$, while the opposite situation prevails in the polar regions resulting in an excess reaching $\sim 25 \%$ near the polar direction. In a sense, the assumption of axi-symmetry leads to transferring electrons from the mid-latitude regions to the polar regions. For a given image of the $\mathrm{K}$-corona, it is not possible to state which geometric assumption is the most appropriate. This would require an a priori knowledge of the three-dimensional distribution of electrons, for instance by stereoscopic imaging or a tomographic reconstruction.

\section{Comparison with previous results}

In this section, we compare our results for the electron density in the solar corona with previously published results. A comparison of preliminary results with earlier measurements has already been published by Lamy et al. (1997). Here, we present a comparison with more recent data.

Kohl et al. (1998) and Cranmer et al. (1999) have used the UVCS White Light Channel measurements to derive polarized brightness values between 1.5 and 4.0 solar radii over polar coronal holes. A least-squares fit to the radial dependence of $p B$ is given by Eq. (2) of Cranmer et al. (1999). The measurements used were taken by UVCS between November 1996 and April 1997. In Fig. 11, we show a comparison of our results obtained for the average of December 1996 with the values published by Cranmer et al. (1999). Two curves have been extracted from Fig. 7, one for the north pole (squares) and one for the south pole (diamonds). The two dotted lines show the uncertainties of the UVCS measurements. We find here a good agreement between the measurements of $\mathrm{LASCO} / \mathrm{C} 2$ and UVCS. 

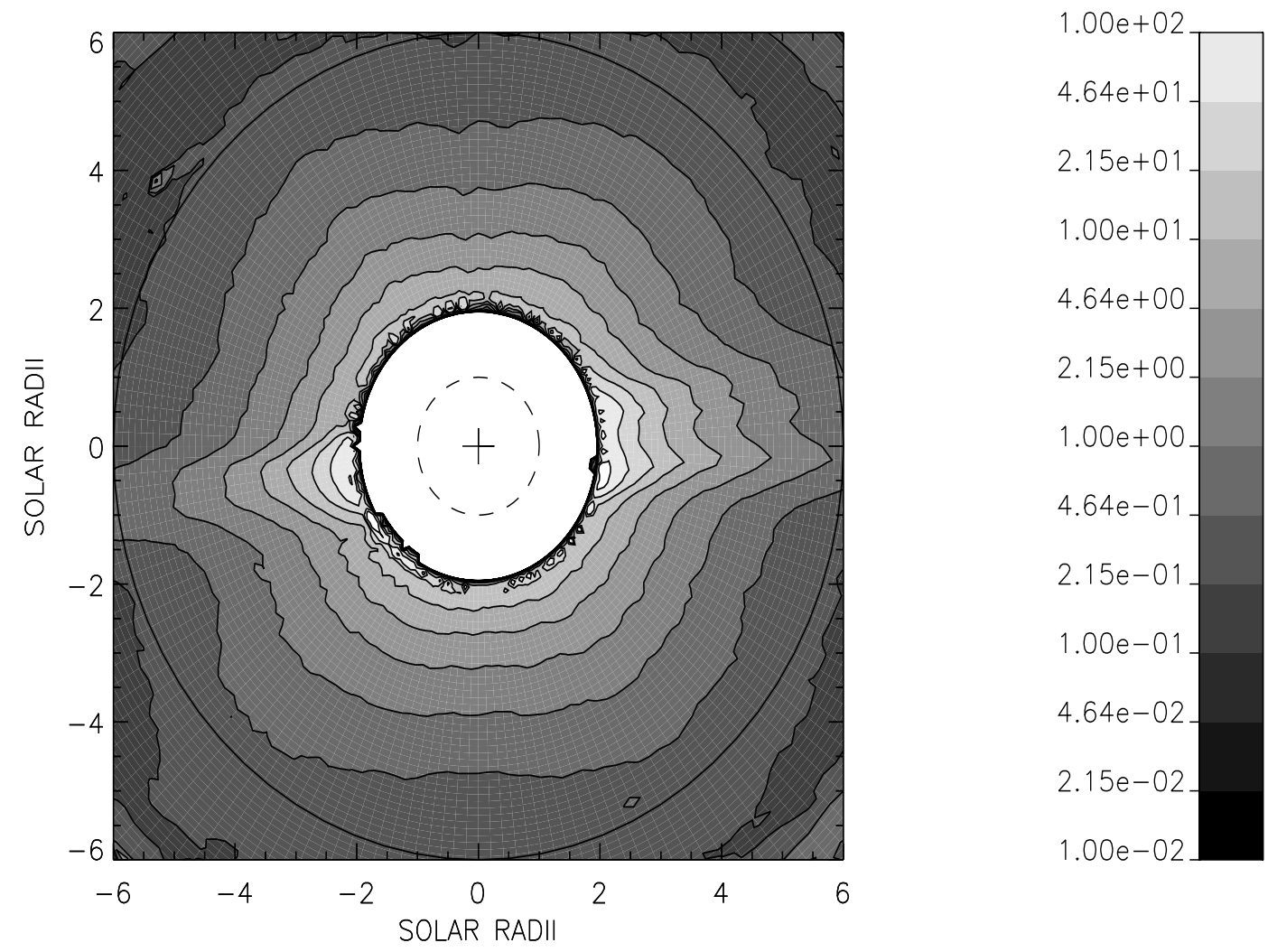

Fig. 3. The white-light image of the K-corona obtained on December 29, 1996. The right-hand scale is in units of $1 . e-10 B_{\odot}$. The solar disk is shown by the dash circle while the outer circle corresponds to $6 R_{\odot}$.
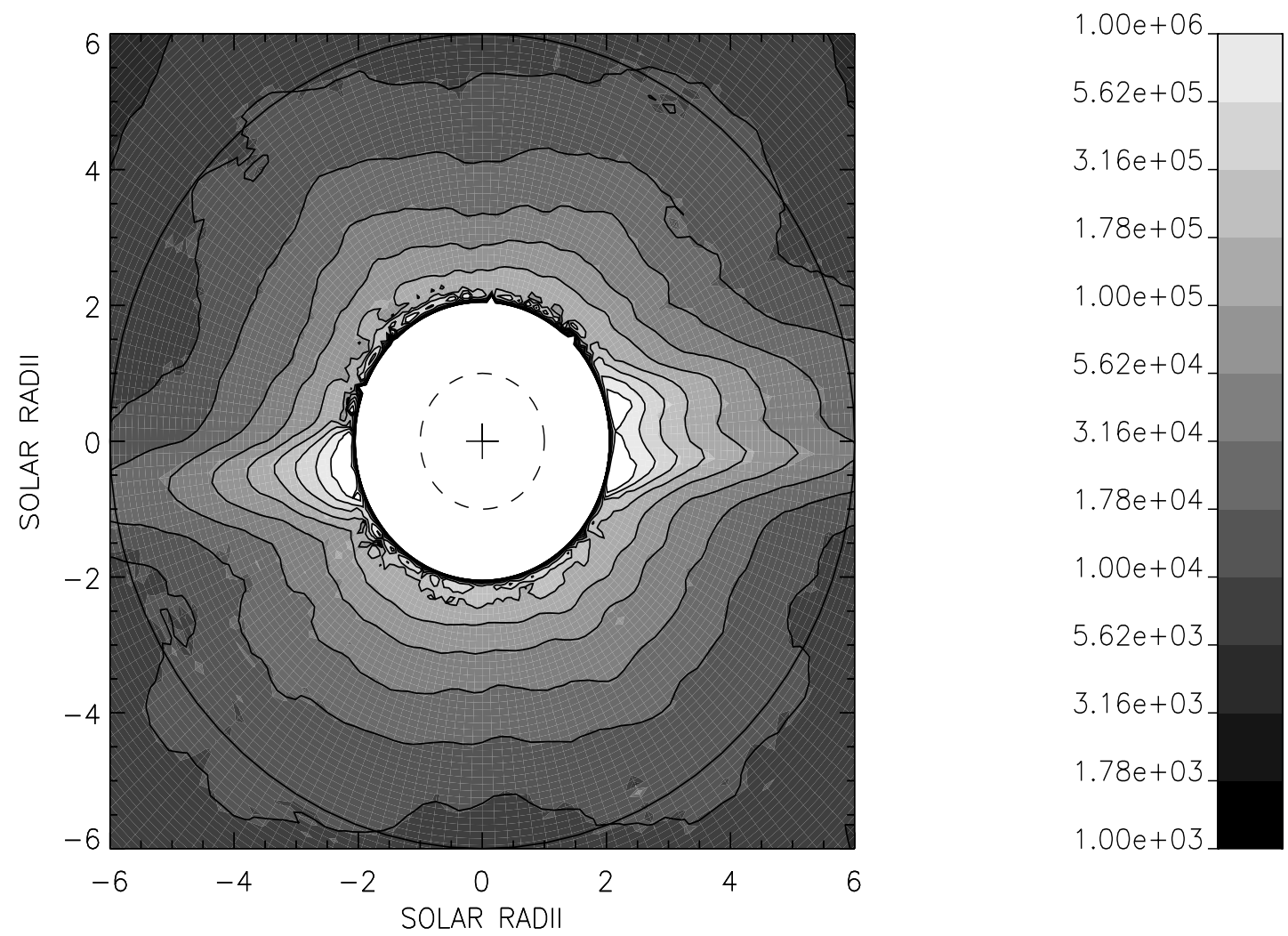

Fig. 4. The $N_{\mathrm{e}}$ map resulting from the inversion of the K-corona shown in the previous figure. The right-hand scale is in units of $\mathrm{cm}^{-3}$.

In the following two figures, we show a comparison of the derived electron density in the corona between 2 and 4 solar radii. Here, we use the results obtained in the case of local spherical symmetry because this is the geometry assumed by 


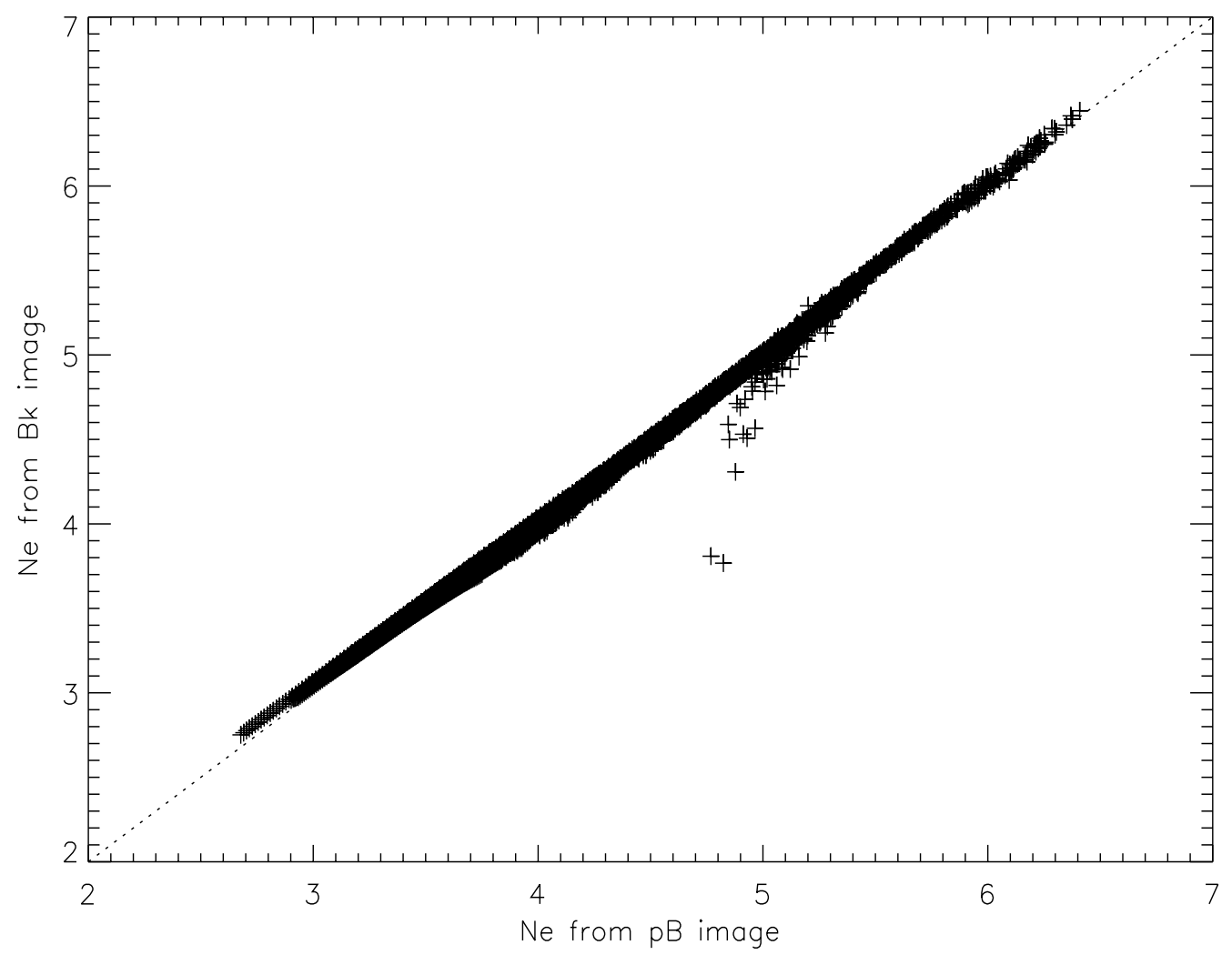

Fig. 5. Regression on a log-log scale between the Ne values obtained from the inversion of the K-corona and those obtained from the $p B$ image obtained on December 29, 1996. The electron density is in $\mathrm{cm}^{-3}$. The dotted line represents a linear fit to the data with a slope equal to 1 .

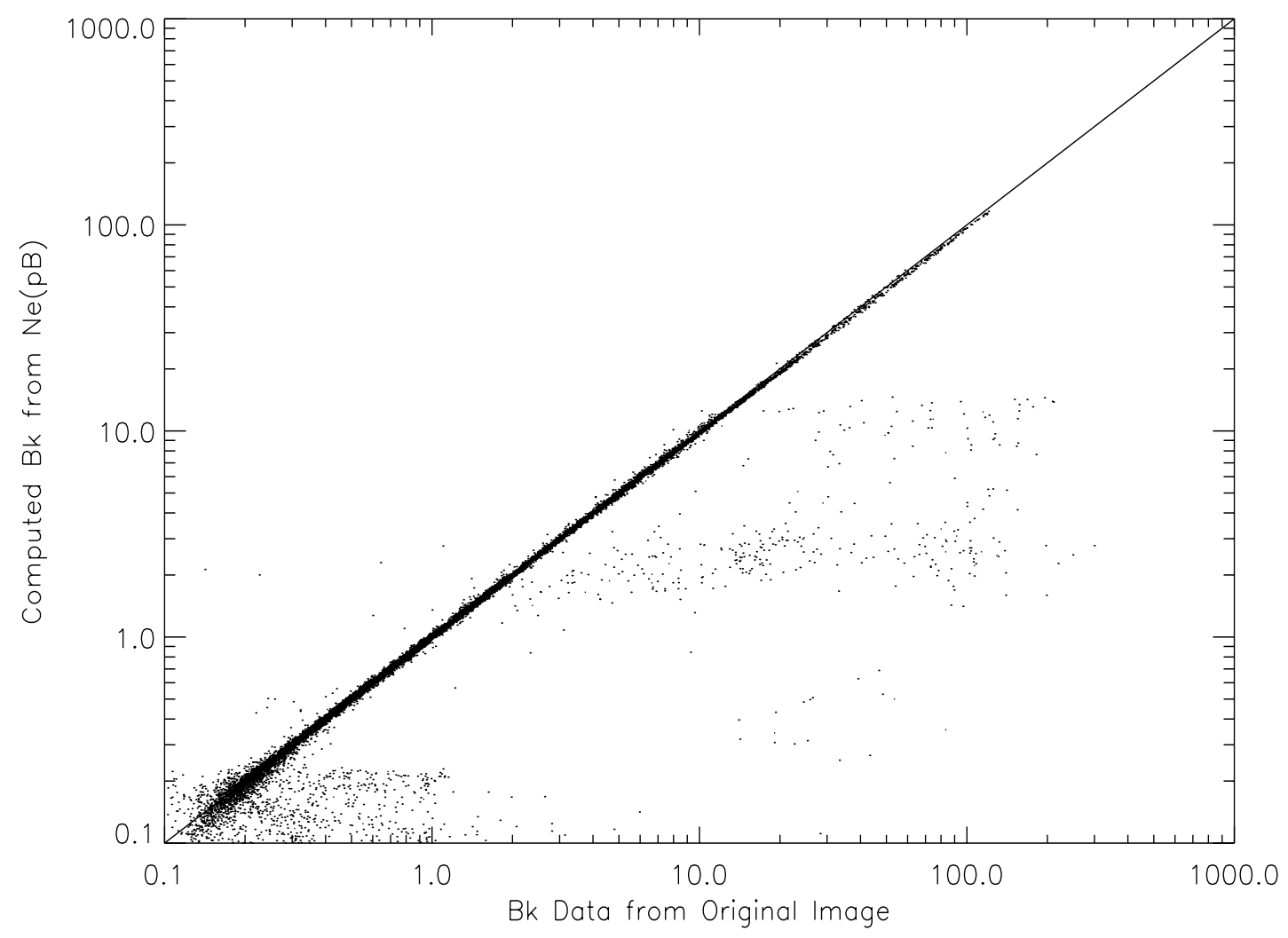

Fig. 6. Regression on a log-log scale between the radiance calculated from the electron density (derived from the $p B$ image) and the original data.The dotted line represents a linear fit to the data with a slope equal to 1 . Note that the computed brightness is smoother than the original data. 

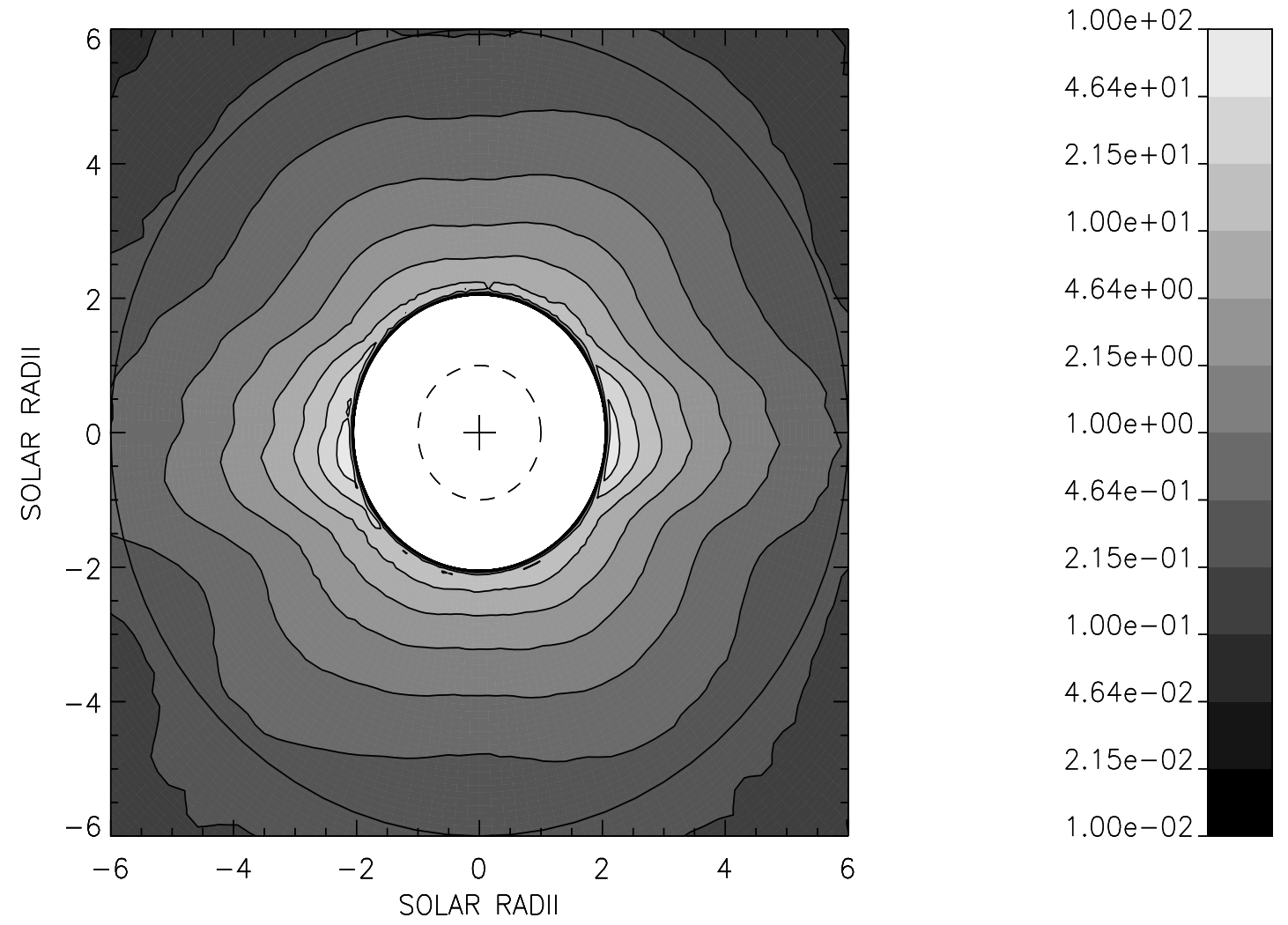

Fig. 7. The average white-light polarized radiance of the solar corona observed in December 1996. The right-hand scale is in units of $1 . e-10 B_{\odot}$. The solar limb is shown by the dashed circle. The solar disk is shown by the dash circle while the outer circle corresponds to $6 R_{\odot}$.
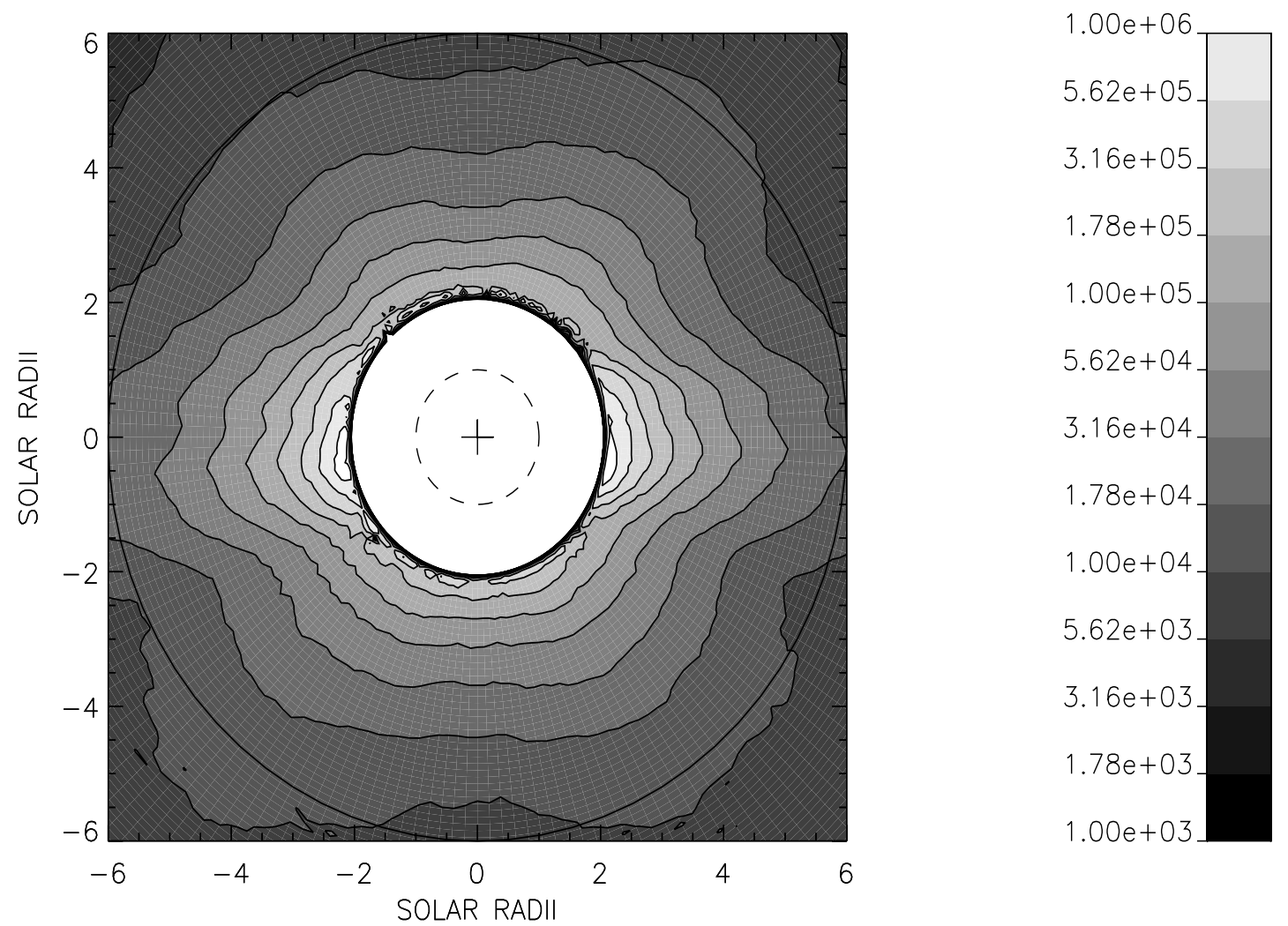

Fig. 8. The $N_{\mathrm{e}}$ map resulting from the inversion of the average white-light polarized radiance of December 1996 under the assumption of local spherical symmetry. The right-hand scale is in $\mathrm{cm}^{-3}$. 

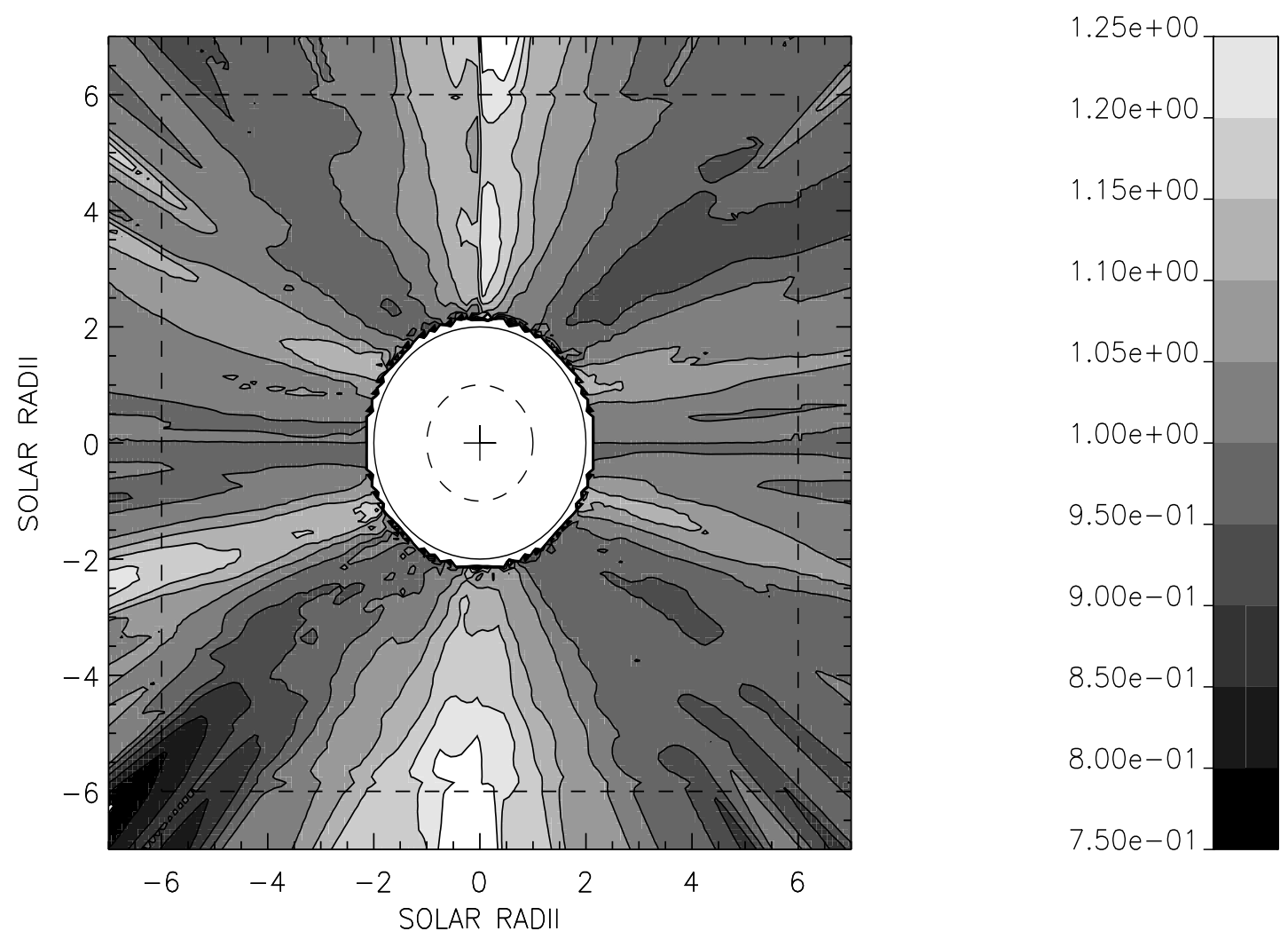

Fig. 9. This image displays the ratio of the electron density obtained under the assumption of axi-symmetry to that obtained under the assumption of spherical symmetry. The right-hand scale displays the values of the ratio. The dash line shows the limit at 6 solar radii.
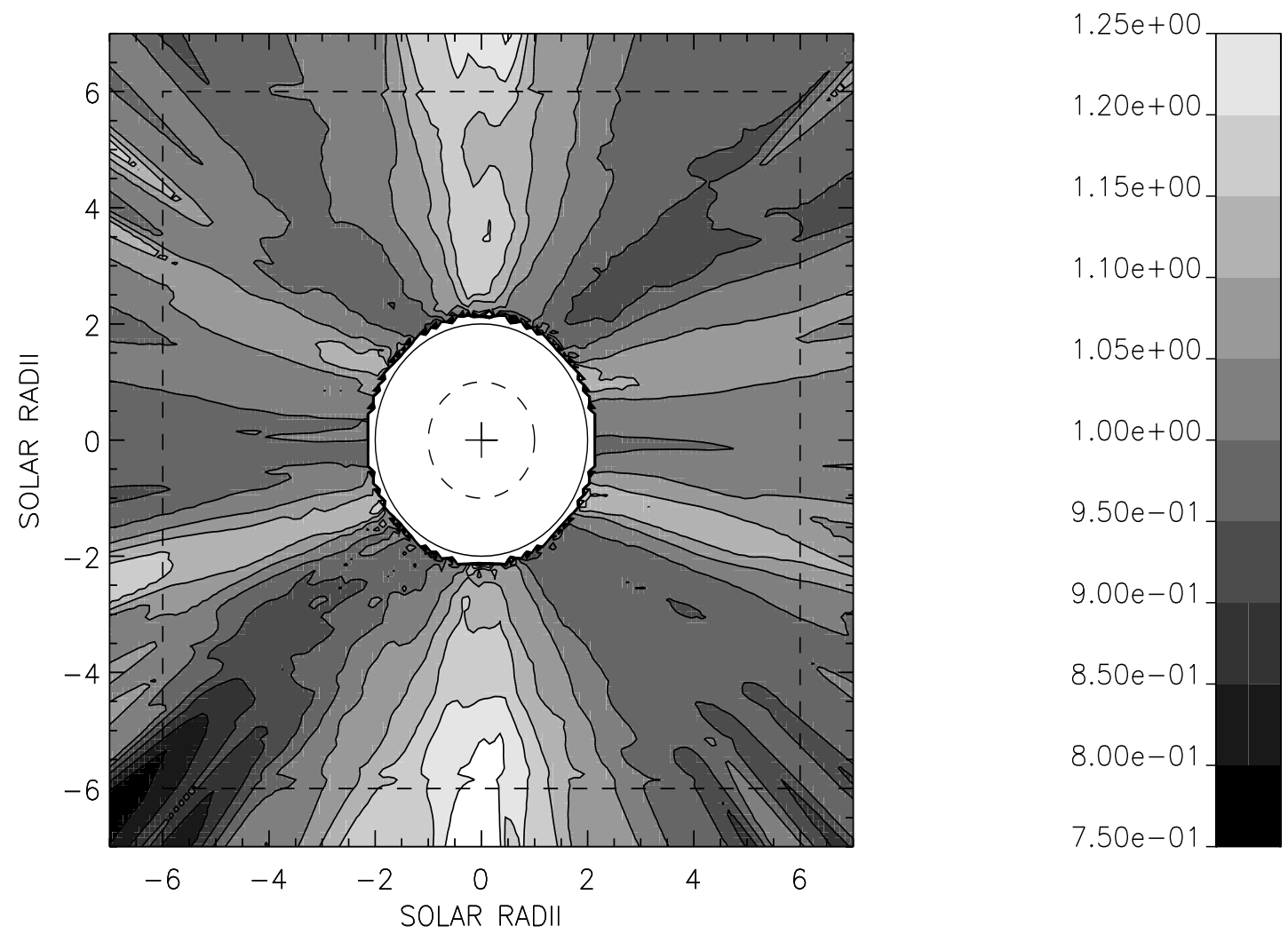

Fig. 10. This image displays the ratio of the electron density obtained under the assumption of quasi axi-symmetry to that obtained under the assumption of spherical symmetry. The right-hand scale displays the values of the ratio. The dash line shows the limit at 6 solar radii. 


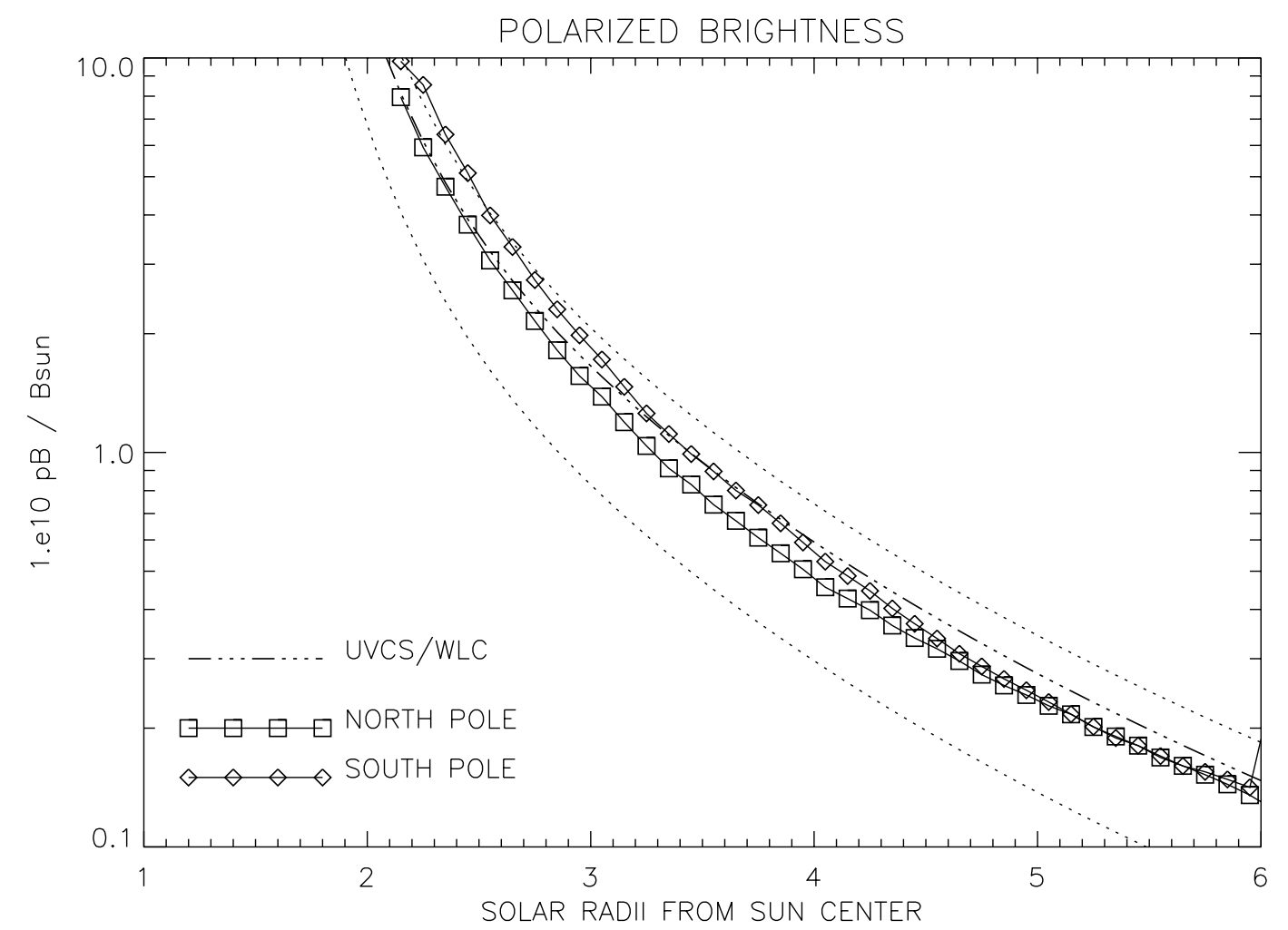

Fig. 11. Comparison of the polarized radiance $p B$ observed by UVCS/WLC and LASCO/C2 in December 1996. The dotted lines give the range of uncertainty for the UVCS/WLC measurements. The radiance is in units of $10^{-10} B_{\odot}$.

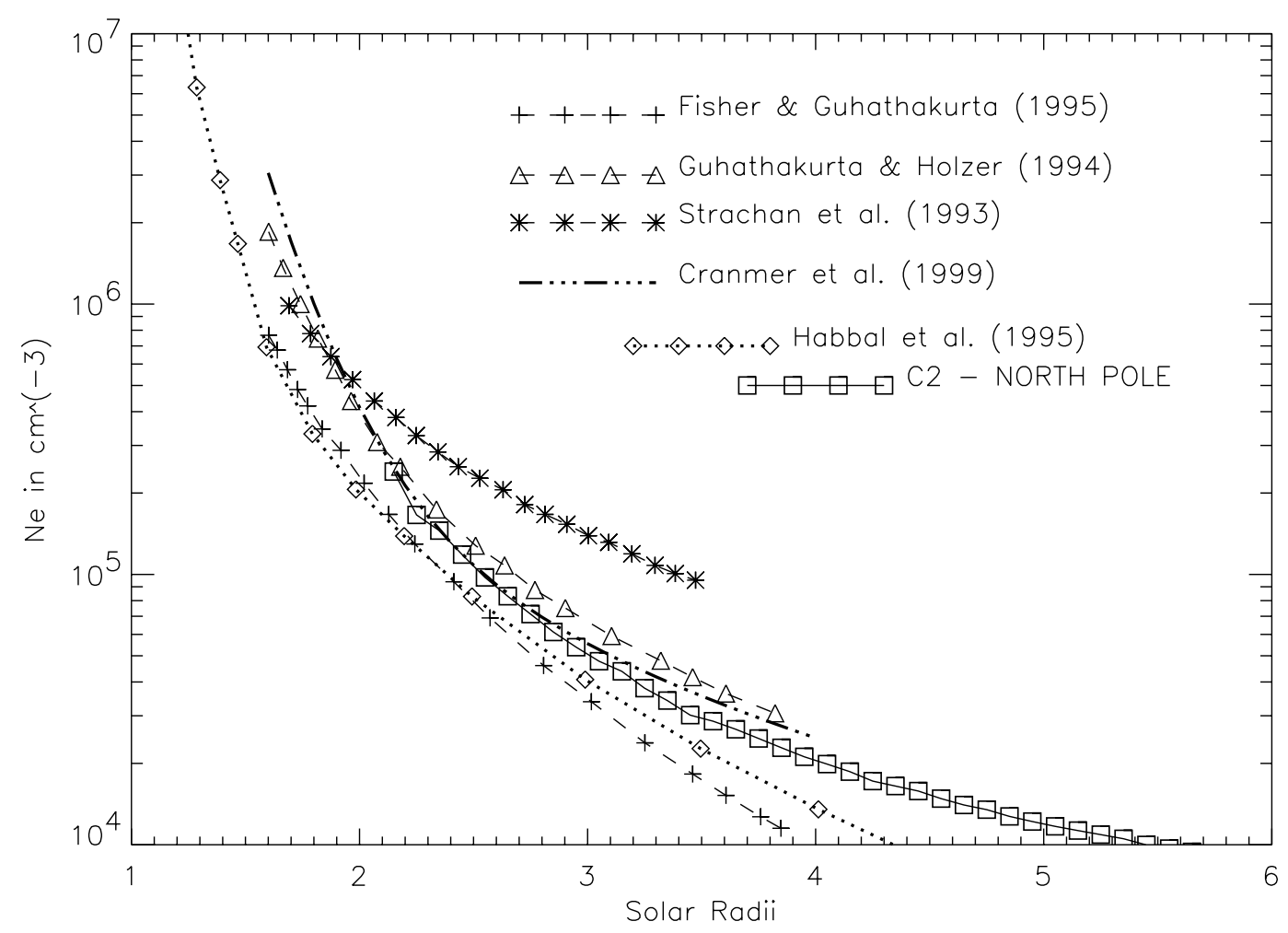

Fig. 12. Comparison of UVCS/WLC and LASCO/C2 electron density values derived from the $p B$ values shown in the previous figure. We have added other measurements by Strachan et al. (1993), Guhathakurta \& Holzer (1994), Fischer \& Guhathakurta (1995) and Habbal et al. (1995).

Cranmer et al. (1999). In Fig. 12, the overall fit between the two sets of data is good. We have also added the results of
Strachan et al. (1993), Guhathakurta \& Holzer (1994) and Fisher \& Guhathakurta (1995) which are also discussed in 
Cranmer et al. (1999). Other radial profiles were published by Habbal et al. (1995). We find a very good agreement with the values given by Guhathakurta \& Holzer (1994).

\section{Conclusion and applications}

In this work, we have presented an algorithm developed to invert the LASCO/C2 white-light images. The algorithm structure can either be applied to polarized brightness images or to K-corona radiance images after proper removal of the stray light and F-corona. We have shown that the results are essentially similar independently of the type of input. For comparison with ground-based observations, it will be easier to use $p B$ images.

We have also compared two types of inversion methods, assuming different global geometries of the solar corona. At times of minimum of solar activity, the cylindrical geometry seems more appropriate. A comparison with the result obtained in the case of the standard local spherical symmetry shows a difference of about $25 \%$ in the electron number density values derived above the solar poles. This will be of interest when applied to actual modeling of the solar coronal density structure.

This inversion method will be applied to the whole set of polarized brightness images obtained by LASCO/C2. The electron density maps will be used in conjunction with other data to derive flow characteristics in the solar corona.

Acknowledgements. SOHO is a mission of international cooperation between ESA and NASA. The LASCO project at Laboratoire d'Astronomie Spatiale is supported by contracts from CNES and by CNRS.

\section{References}

Allen, C. W. 1973, Astrophysical quantities, third edition, University of London (The Athlone Press)

Altschuler, M. D., \& Perry, R. M. 1972, Sol. Phys., 23, 410

Billings, D. E. 1966, A Guide to the Solar Corona (New York Academic Press)

Brueckner, G. E., Howard, R. A., Koomen, M. J., et al. 1995, Sol. Phys., 162, 357

Cranmer, S. R., Kohl, J. L., Noci, G., et al. 1999, ApJ, 511, 481

Crifo-Magnant, F., \& Picat, J. P. 1980, A\&A, 88, 97

Fisher, R., \& Guhathakurta, M. 1995, ApJ, 447, L139

Gabryl, J.-R., Cugnon, P., \& Clette, F. 1999, Solar Wind Nine, AIP Conf. Proc., 471, 749

Guhathakurta, M., \& Holzer, T. E. 1994, ApJ, 426, 782

Habbal, S. R., Esser, R., Guhathakurta, M., \& Fisher, R. R. 1995, Geophys. Res. Lett., 22, 1465

Hayes, A. P., Vourlidas, A., \& Howard, R. A. 2001, ApJ, 548, 1081

Lamy, P. L., et al. 1997, Electronical Densities in Coronal Holes from LASCO-C2 Images, ESA SP-404, 491

Lamy, P., Llebaria, A., \& Quemerais, E. 2002, Adv. Space Res., 29, 373

Llebaria, A., Lamy, P. L., \& Koutchmy, S. 1999, The Global Activity of the Solar Corona, ESA SP-446, 441

Minnaert, M. 1930, Zs. Ap., 1, 209

Munro, R. H., \& Jackson, B. V. 1977, ApJ, 213, 874

Strachan, L., Kohl, J. L., Weiser, H., Withbroe, G. L., \& Munro, R. H. 1993, ApJ, 412, 410

Saito, K. 1950, A Non Spherical Axisymmetric Model of the Solar KCorona of the Minim um Type, Ann. Tokyo Astron. Obs. Vol. III1,53

Van de Hulst, H. C. 1950, The Electron Density of the Solar Corona, Bull. Astron. Inst. Neth., 11, 135 